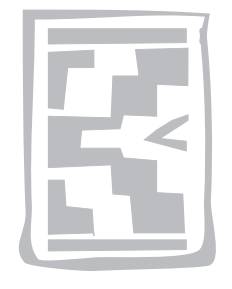

\title{
Electrocardiographic surrogates of structural myocardial alterations in the Dorper sheep heart
}

\author{
J. KER ${ }^{1}$ and E.C. WEBB ${ }^{2}$
}

\begin{abstract}
KER, J. \& WEBB, E.C. 2005. Electrocardiographic surrogates of structural myocardial alterations in the Dorper sheep heart. Onderstepoort Journal of Veterinary Research, 72:273-277

In this study we evaluated the validity of well-known human electrocardiographic markers of myocardial pathology in Dorper sheep. These markers include: the duration of the QRS complex of premature ventricular complexes (PVCs), the presence of notching of the QRS complex of PVCs and change of the ST-segment of PVCs. It was shown that these three electrocardiographic phenomena correlate with myocardial pathology in the hearts of Dorper sheep. We also describe a new electrocardiographic indicator of myocardial pathology, namely an increase in the frequency of cardiac memory T waves as a new electrocardiographic surrogate for myocardial pathology in the hearts of Dorper sheep.

Keywords: Cardiac memory T waves, Dorper sheep, electrocardiography, myocardial pathology, premature ventricular complexes
\end{abstract}

\section{INTRODUCTION}

It has been shown that certain characteristics of a premature ventricular complex (PVC) can reflect either the presence or the absence of myocardial disease (Soloff 1961; Schamroth 1980; Moulton, Medcalf \& Lazzara 1990). A PVC is the expression of an impulse that arises prematurely in an ectopic focus and can originate in the specialized conduction tissue distal to the bifurcation of His or in the ventricular myocardium itself (Soloff 1961; Myerburg \& Kessler 1998). On the 12-lead, surface electrocardiogram (ECG) PVCs are recognized by the

1 Department of Physiology, University of Pretoria and Pretoria Heart Hospital, P.O. Box 24318, Gezina, Pretoria, 0031 South Africa. E-mail: jker@wol.co.za

2 Department of Animal and Wildlife Sciences, Faculty of Agricultural Sciences, University of Pretoria, Pretoria, 0002 South Africa

Accepted for publication 14 June 2005-Editor premature occurrence of a QRS complex that is abnormal in shape and with a duration that exceeds that of the dominant QRS complex. The T wave of a PVC is opposite in direction to the major deflection of the QRS complex (Myerburg \& Kessler 1998). However, narrow PVCs can occur and have been explained as originating at a point equidistant from each ventricle in the ventricular septum or by arising high in the fascicular system (Olgin \& Zipes 2001). Currently, three mechanisms of PVC generation are recognized: enhanced automaticity, triggered activity and re-entry (Lerman 2000).

PVCs originating in normal myocardium where no pathology is present, have the following characteristics (Soloff 1961; Schamroth 1980):

(a) The QRS amplitude is large and exceeds 20 $\mathrm{mm}$, or higher.

(b) The QRS complex does not exceed $120 \mathrm{~ms}$ in duration.

(c) The QRS deflection has a smooth contour with no notching 
(d) The ST-segment and T wave are opposite in direction to the QRS deflection.

(e) The ST-segment does not display any isoelectric period.

(f) The ST-segment blends imperceptibly with the proximal limb of the $T$ wave, so that the two cannot be separated.

(g) The $T$ wave has asymmetrical limbs.

Myocardial pathology can alter these features of "uncomplicated" PVCs and any one or more of the following changes are indicative of "complicated" PVCs, where "complicated" is indicative of the presence of myocardial pathology (Soloff 1961; Schamroth 1980):

(a) The QRS complex is diminished in amplitude.

(b) The QRS complex widens and exceeds 120 $\mathrm{ms}$.

(c) There is marked notching and irregularity of the QRS complex.

(d) The ST-segment displays an isoelectric period.

(e) The $T$ waves tend to be sharply pointed and symmetrical.

(f) The T wave has the same polarity as that of the QRS complex.

However, the above data is applicable to the human heart and no such data exists for the Dorper sheep heart. In addition, we wanted to know whether cardiac memory $T$ waves can be used as another electrocardiographic indicator of myocardial pathology.

Cardiac memory is an electrocardiographic phenomenon reflected in the $T$ wave, when $T$ waves of normally conducted beats seem to "remember" the polarity of the QRS complexes of previous abnormally conducted beats (Rosenbaum \& Blanco 1982; Rosen 2001). Currently, it is still unclear whether cardiac memory $T$ waves can be used as an electrocardiographic warning for myocardial pathology to come (Rosen 2001).

We have a valid model for the induction of PVCs and cardiac memory in the Dorper sheep heart (Ker \& Webb 2003; Ker, Webb, Ker \& Bekker 2003) and the purpose of this study was to examine the possibility that cardiac memory $T$ waves can be used as an electrocardiographic indicator of myocardial pathology and secondly, whether the characteristics of "complicated" PVCs are true for the Dorper sheep heart.

\section{MATERIALS AND METHODS}

This study was performed with the approval of and adherence to the guidelines of the Pretoria Bio- medical Research Centre's Animal Use and Care Committee.

In this study, we looked at existing electrocardiographic tracings on ten Dorper wethers that were used in our experiments on cardiac memory $T$ waves induced by PVCs, and the structural effects of PVCs on myocardial histology (Ker, Webb, Ker \& Bekker 2003; Ker, Webb \& Van der Merwe 2004). In these experiments, PVCs were induced by the guidewire of an Arrow central venous pressure catheter set that was advanced into the right ventricle via the left internal jugular vein by the Seldinger technique. PVCs induced in this way are capable of inducing cardiac memory $T$ waves and furthermore, it has been shown that Dorper hearts exposed to prolonged periods of PVCs in this way develop structural abnormalities of their left ventricles, the ventricle not exposed to the guidewire (Ker, Webb \& Van der Merwe 2004). Whether these left ventricular, myocardial alterations are caused by the PVCs themselves, by the guide-wire, infection, or some other, unknown culprit is not relevant for this study, because we wanted to document changes in the morphology of PVCs, compared between normal and abnormal left ventricles. In this way every wether serves as its own control in the following way: At the start of the study when the left ventricle is structurally normal, the character of PVCs and/or memory $T$ waves should differ from those at the end of the study when the left ventricles are structurally abnormal (the cause of these changes is not relevant, as we wanted to correlate electrocardiographic changes with histologic changes).

\section{RESULTS}

The ten wethers were exposed to PVCs for a mean number of 21 days.

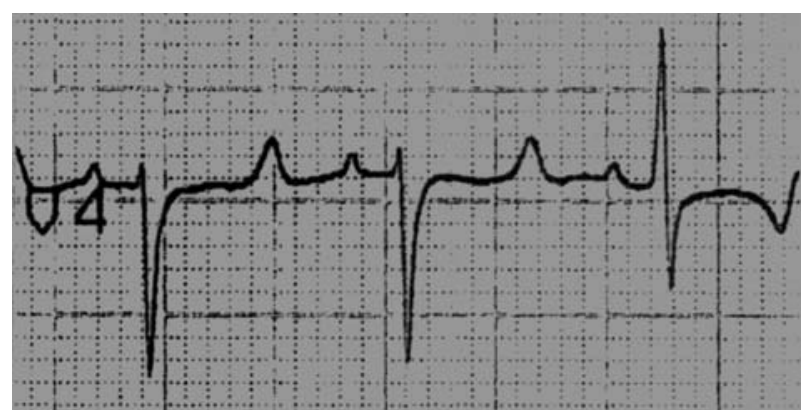

FIG. 1 An example of an uncomplicated PVC occurring in the normal ovine heart during the first day of being subjected to PVCs. The third beat in this tracing from lead V4 is a PVC. Note the narrow QRS complex with a duration of $40 \mathrm{~ms}$ and the isoelectric ST segment. No notching of the QRS complex is present 
Three morphological changes were noted in PVCs when comparing the first with the last day of study. First, the QRS duration of the PVCs increased. Secondly, notching appeared in the QRS complexes of the PVCs. Lastly, the isoelectric ST-segments of the PVCs disappeared (see Table 1). See Fig. 1 for an example of an uncomplicated PVC and Fig. 2 as an example of a PVC displaying these morphological alterations.

In order to detect if there is any difference between the early and late occurrence of cardiac memory $T$ waves, the first and last $10 \%$ of PVCs were evaluated in every wether (Table 2). The T wave of the first normal beat after every PVC were evaluated in order to determine whether these $T$ waves retained the vector of the previous PVC QRS complex (see Fig. 3).

Histological evaluation of the left ventricles revealed myocarditis in all the specimens, based on myocytolysis and an infiltration of white blood cells into the myocardium (see Fig. 4 and 5) (Ker, Webb \& Van der Merwe 2004).

TABLE 1 Electrocardiographic characteristics of PVCs on the first and last day of study

\begin{tabular}{|c|c|c|c|c|c|c|c|c|}
\hline $\begin{array}{l}\text { Sheep } \\
\text { number }\end{array}$ & $\begin{array}{l}\text { Mean QRS } \\
\text { duration } \\
\text { on first day } \\
\text { (ms) }\end{array}$ & $\begin{array}{l}\text { Mean QRS } \\
\text { duration } \\
\text { on last day } \\
\text { (ms) }\end{array}$ & $\begin{array}{l}\text { Number of } \\
\text { PVCs } \\
>0.06 \mathrm{~s} \\
\text { on first day }\end{array}$ & $\begin{array}{l}\text { Number of } \\
\text { PVCs } \\
>0.06 \mathrm{~s} \\
\text { on last day }\end{array}$ & $\begin{array}{l}\text { Number of } \\
\text { PVCs with } \\
\text { notching } \\
\text { on first } \\
\text { day }\end{array}$ & $\begin{array}{l}\text { Number of } \\
\text { PVCs with } \\
\text { notching } \\
\text { on last day }\end{array}$ & $\begin{array}{l}\text { Number of } \\
\text { PVCs with } \\
\text { isoelectric } \\
\text { ST- } \\
\text { segments } \\
\text { on first day }\end{array}$ & $\begin{array}{l}\text { Number of } \\
\text { PVCs with } \\
\text { isoelectric } \\
\text { ST- } \\
\text { segments } \\
\text { on last day }\end{array}$ \\
\hline 1 & 0.038 & 0.060 & 20 & 20 & 2 & 2 & 44 & 44 \\
\hline 2 & 0.040 & 0.070 & 1 & 15 & 0 & 9 & 13 & 2 \\
\hline 3 & 0.042 & 0.080 & 8 & 48 & 3 & 3 & 76 & 76 \\
\hline 4 & 0.040 & 0.060 & 4 & 16 & 1 & 10 & 13 & 3 \\
\hline 5 & 0.038 & 0.080 & 4 & 18 & 1 & 11 & 15 & 4 \\
\hline 6 & 0.040 & 0.060 & 2 & 10 & 1 & 7 & 11 & 2 \\
\hline 7 & 0.038 & 0.070 & 32 & 130 & 9 & 81 & 110 & 22 \\
\hline 8 & 0.040 & 0.070 & 3 & 18 & 2 & 9 & 14 & 3 \\
\hline 9 & 0.038 & 0.060 & 3 & 13 & 1 & 8 & 15 & 3 \\
\hline 10 & 0.038 & 0.080 & 3 & 30 & 3 & 22 & 25 & 5 \\
\hline
\end{tabular}

Increase in QRS duration:
$P=0.013$ (paired t-test). OR $=13.93$ (Odds ratio that the QRS duration of PVCs will increase from $<0.06 \mathrm{~s}$ on the first day of study to $>0.06 \mathrm{~s}$ on the last day of study)

\section{Notching of QRS complexes: \\ QRS complexes on the last day of study)}

Disappearance of isoelectric ST-segments: $P=0.031$ (paired t-test). OR $=12.86$ (Odds ratio that the isoelectric ST-segment of PVCs will disappear on the last day of study)

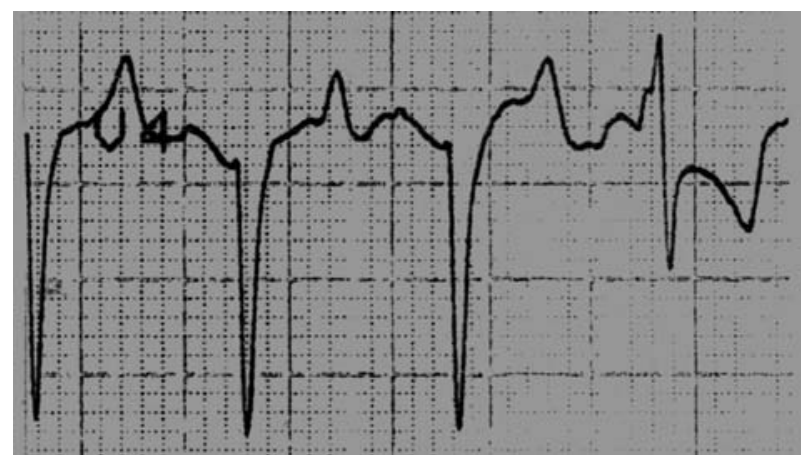

FIG. 2 The tracing was taken from lead V4 from the same sheep's tracing illustrated in Fig. 1, but this time with 14 days of exposure to PVCs. The changes are possibly indicative of myocardial pathology. The third beat is a PVC. Note the notching of the QRS complex. The QRS complex is much broader than that illustrated in Fig. 1, with a duration of $80 \mathrm{~ms}$. Also note the loss of the ST segment
TABLE 2 Early versus late cardiac memory T waves

\begin{tabular}{|l|l|l|}
\hline $\begin{array}{l}\text { Sheep } \\
\text { number }\end{array}$ & $\begin{array}{l}\text { Number of early } \\
\text { memory T waves }\end{array}$ & $\begin{array}{l}\text { Number of late } \\
\text { memory T waves }\end{array}$ \\
\hline 1 & 2 & 5 \\
2 & 17 & 21 \\
3 & 2 & 3 \\
4 & 8 & 12 \\
5 & 15 & 15 \\
6 & 8 & 37 \\
7 & 40 & 188 \\
8 & 10 & 21 \\
9 & 31 & 90 \\
10 & 40 & 51 \\
\hline
\end{tabular}

$$
P=0.049 \text { (paired t-test) }
$$

$\mathrm{OR}=10.38$ (Odds ratio that there will be an increase in frequency of cardiac memory $T$ waves between the first and last day of study) 


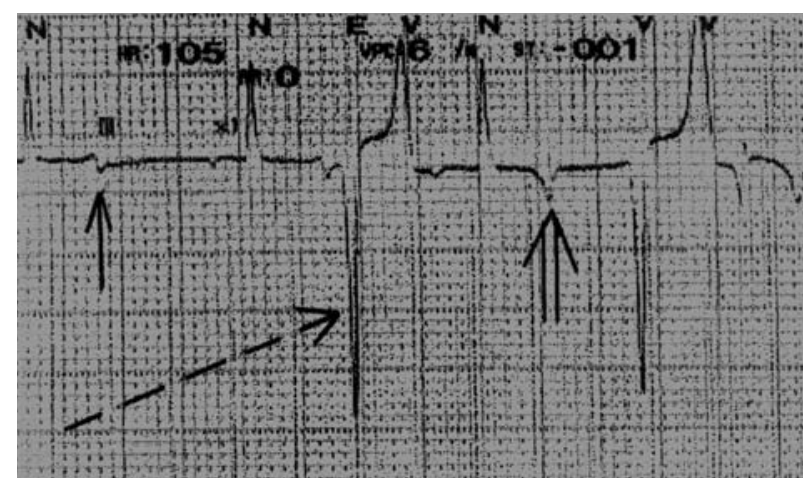

FIG. 3 An example of cardiac memory T waves. The third and fifth beats in this tracing are PVCs (broken arrow). Note the bifid T waves before the first PVC (arrow), and the inverted T waves after the PVCs (double arrow). The T wave retains the vector of the QRS complex of the PVC, hence the name "cardiac memory"

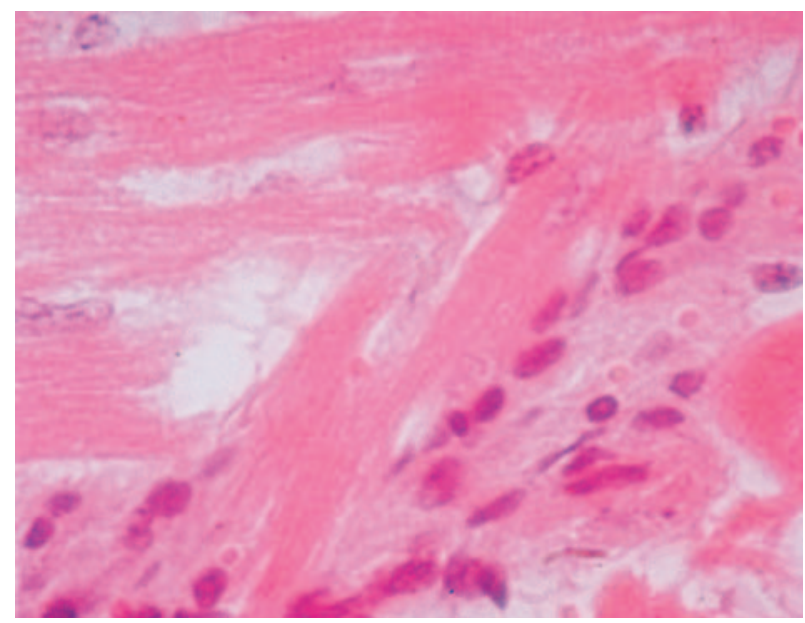

FIG. 5 Myocytolysis, another feature of myocarditis, occurs in addition to the inflammatory cell infiltrate in the left ventricle

\section{DISCUSSION}

On the first day of PVC exposure in the wethers the PVCs had the following characteristics:

(a) The QRS duration did not exceed $60 \mathrm{~ms}$.

(b) There was no notching of the QRS complex.

(c) The ST-segment was isoelectric.

On the last day of PVC exposure certain changes were noted in the PVCs:

(a) The QRS duration prolonged to $>60 \mathrm{~ms}$

(b) Notching of the QRS complex appeared.

(c) The isoelectric ST-segment disappeared.

It is important to realize that normal Dorper wethers were used in these studies. Six normal wethers were slaughtered and their left ventricles were subjected to histological examination in order to docu-

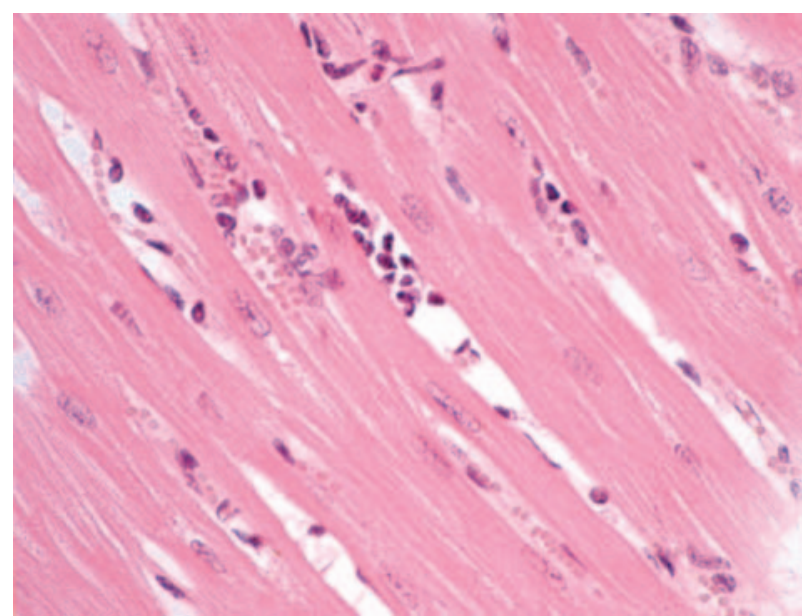

FIG. 4 Infiltration of the left ventricular interstitium by mixed inflammatory cells, a feature of myocarditis

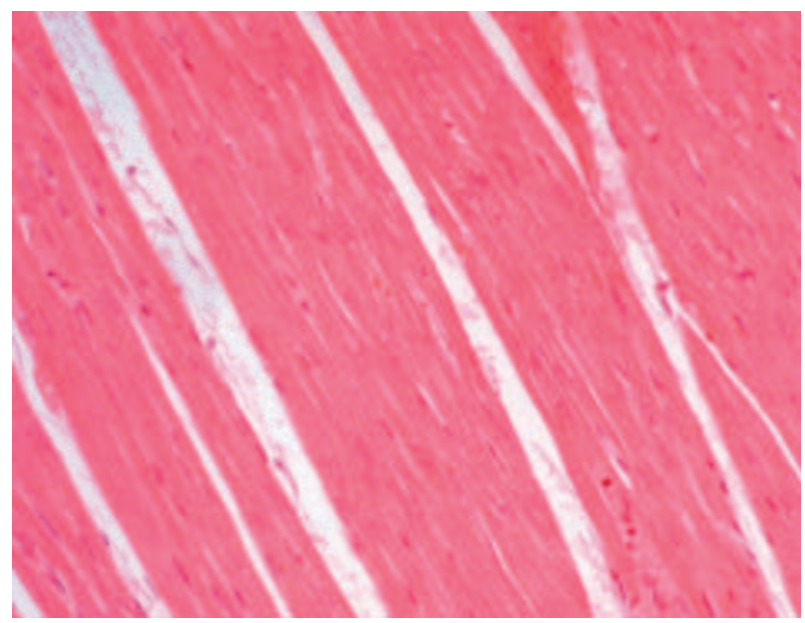

FIG. 6 Normal Dorper heart muscle

ment the normal histological appearance of the Dorper sheep heart (see Fig. 6). At the end of these studies all the wethers which had been subjected to PVCs for a mean of 21 days, had an abnormal histological appearance of the left ventricle, indicative of myocarditis. In this way every wether served as its own control-at the beginning of the study when normal wethers entered the study, the PVCs had different characteristics than at the end of the study when myocardial pathology was present. As we want to document that certain changes occurs in PVCs when the left ventricle undergoes structural alteration, the specific cause of these structural changes is not relevant.

It is concluded that the following characteristics of PVCs can be used to identify underlying myocardial pathology in the Dorper sheep heart: 
(a) The QRS duration will exceed $60 \mathrm{~ms}$.

(b) Notching of the QRS complex.

(c) Disappearance of the ST-segment.

In addition, we identified a new electrocardiographic surrogate for myocardial pathology-the cardiac memory $T$ wave. It is shown in this study that the true value of using cardiac memory $T$ waves as an electrocardiographic surrogate for structural myocardial alterations in the Dorper heart does not lie in an instantaneous electrocardiographic assessment, but in electrocardiographic follow-up, in order to determine if there is an increase in the frequency of cardiac memory $\mathrm{T}$ waves. As shown in this study, an increase of at least $42 \%$ in the frequency of cardiac memory $T$ waves, following PVCs is indicative of underlying structural myocardial alterations. Although al four PVC changes were statistically significant with $P$ values below 0.05 , the increase in QRS duration had the lowest $P$ value $(=0.013)$ and the increase in cardiac memory $T$ wave frequency had the highest $P$ value $(=0.049)$.

An unexplained phenomenon is the disappearance of the isoelectric ST-segments of PVCs at the end of the study, when myocardial pathology is present. This is in contrast to the human heart, where it has been described that the appearance of isoelectric ST-segments is a sign of myocardial pathology. This observation is consistent, but unexplained and will need clarification in future studies. It is possible that some metabolic difference between the human and ovine heart may offer an explanation to this observation.

\section{REFERENCES}

KER, J. \& WEBB, E.C. 2003. Ventriculo-atrial conduction in the ovine heart, caused by premature ventricular complexes. Onderstepoort Journal of Veterinary Research, 70:107-111.

KER, J., WEBB, E.C., KER, J.A. \& BEKKER, P.A. 2003. The heart remembers: observations of cardiac memory in the Dorper sheep heart. Onderstepoort Journal of Veterinary Research, 70:299-305.

KER, J., WEBB, E.C. \& VAN DER MERWE, C.F. 2004. Ventricular dyssynchrony as a cause of structural disease in the heart of Dorper sheep. Onderstepoort Journal of Veterinary Research, 71:197-202.

LERMAN, B.B. 2000. Ventricular arrhythmias and sudden death, in Cecil textbook of medicine, $21^{\text {st }}$ ed., edited by B.B. Lerman. Philadelphia: W.B. Saunders.

MOULTON, K.P., MEDCALF, T. \& LAZZARA, R. 1990. Premature ventricular complex morphology. A marker for left ventricular structure and function. Circulation, 81:1245-1251.

MYERBURG, R.J. \& KESSLER, K.M. 1998. Recognition, clinical assessment and management of arrhythmias and conduction disturbances, in Hurst's the heart, $9^{\text {th }}$ ed., edited by R.W. Alexander, R.C. Schlant \& V. Fuster. New York: McGrawHill.

OLGIN, J.E. \& ZIPES, D.P. 2001. Specific arrhythmias: Diagnosis and treatment, in Heart disease. A textbook of cardiovascular medicine, $6^{\text {th }}$ ed., edited by E. Braunwald, D.P. Zipes \& P. Libby. Philadelphia: W.B. Saunders.

ROSEN, M.R. 2001. The heart remembers: clinical implications. Lancet, 357:468-471.

ROSENBAUM, M.B. \& BLANCO, H.H. 1982. Electrotonic modulation of the T wave and cardiac memory. American Journal of Cardiology, 50:213-222.

SCHAMROTH, L. 1980. Ventricular extrasystoles, ventricular tachycardia and ventricular fibrillation: Clinical electrocardiographic considerations. Progress in Cardiovascular Diseases, 23:13-32.

SOLOFF, L.A. 1961. Ventricular premature beats diagnostic of myocardial disease. American Journal of Medical Science, 242:315-319. 